\title{
Xenon arc photocoagulation for treatment of subluxation of lens
}

\author{
P A LAMBA, D SANTOSH KUMAR, AND ANITA ARORA \\ From the Department of Ophthalmology, Jawaharlal Institute of Postgraduate Medical Education and \\ Research, Pondicherry, India
}

SUMMARY In the treatment of congenital or acquired subluxation of the lens a conservative approach to reposition the pupil in front of the aphakic area by the use of the xenon arc photocoagulator is emphasised. The management of two cases of subluxation is discussed in detail and the results evaluated. The ease of repositioning the pupil by means of iris photocoagulation and the absence of surgical complications are indications for the use of this non-invasive procedure in the treatment of subluxated lenses.

Subluxation of the lens, congenital or acquired, results in disturbance of vision for various reasons such as uncorrectable lenticular astigmatism, lens opacifications, or the complications of subluxation itself. Uncomplicated subluxation occurs especially in younger people, and when the lens is clear it is usually treated conservatively. The indications for surgical intervention and the choice of procedure are still a matter of controversy.

Surgical techniques previously used in the management of subluxated lenses include iridectomy, discission, aspiration and lens extraction with forceps, and vectis or cryo extraction. Despite recent advances in surgical techniques and skills, the removal of the lens is fraught with a high incidence of operative and postoperative complications ${ }^{12}$ such as vitreous loss, retinal detachment, glaucoma, and intraocular infection. Opening up of the globe to perform an optical

Correspondence to Professor P A Lamba, Lady Hardinge Medical College, New Delhi-110001, India.
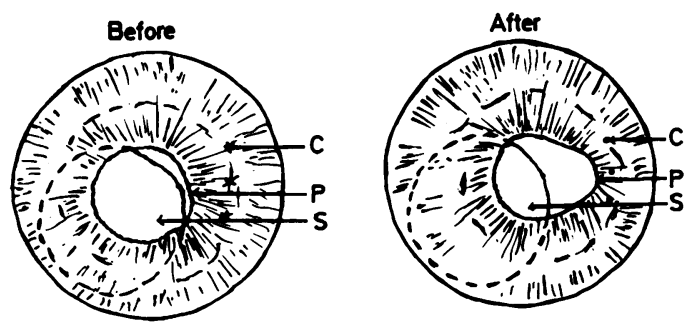

Fig. 1 Before and after coagulation of the iris with xenon arc. $P=$ pupillary border. $S=$ subluxated lens. $C=$ site of coagulation. iridectomy also has the same disadvantages. Straatsma et al. ${ }^{3}$ were the first to carry out iris photocoagulation for improving the visual acuity in cases of subluxation of lens by utilising the aphakic optical system. Peyman et al. ${ }^{4}$ treated 10 eyes with subluxation of the soft lens by the vitrophage, with satisfactory improvement in vision. We now report the results of two such cases managed by iris photocoagulation to reposition the pupil so as to increase the aphakic pupillary zone.

\section{Material and methods}

A xenon arc photocoagulator Clinitex Log 3 was used. Fig. 1 illustrates the principle and the site of iris which is recommended for photocoagulation. This must avoid damage to the sphincter pupillae muscle. The energy to be delivered to iris tissue is minimal and does not produce any iritis. The increased cellularity of the aqueous can be effectively controlled in 24-48 hours with local corticosteroid drops.

\section{Case reports}

CASE 1

A 29-year-old woman attended the Ophthalmology Outpatient Department of Jipmer Hospital in April 1983 with complaints of diminished vision associated with uniocular diplopia in the right eye of three months' duration. In the left eye she had pain and redness five months previously and had undergone lens extraction with no significant visual improvement. 


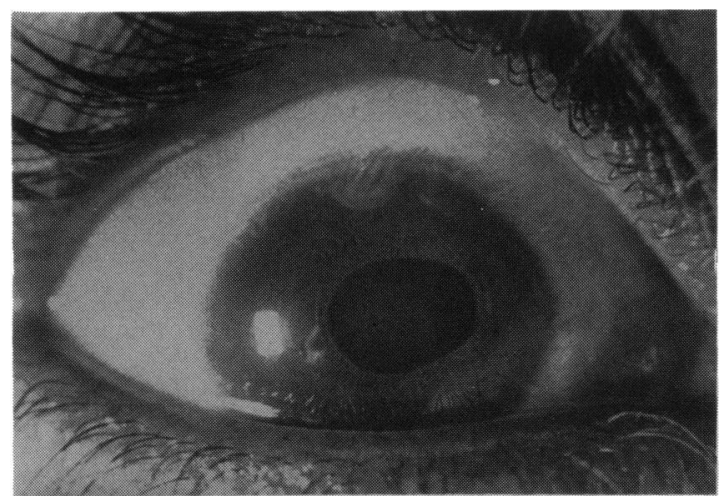

Fig. 2 Repositioning of pupil nasally to enlarge the aphakic area.

Physical examination revealed arachnodactyly and high arched palate. The arm span was $5 \mathrm{~cm}$ more than the height. However, there were no cardiovascular abnormalities. Ocular examination showed that the right eye was quiet. The anterior chamber was deep and slightly irregular with iridodonesis. The pupil was normal in size and reaction to light. The lens was partly cataractous, and visual acuity was finger counting at 1 metre. On dilatation of the pupil the lens was found to be subluxated laterally and slightly downwards, and aphakic correction afforded substantial improvement of visual acuity. In the left eye the cornea showed bullous keratopathy with pigment on posterior surface. Aphakia with herniation of vitreous into the anterior chamber was present. The IOP was $50.4 \mathrm{mmHg}$ (Schiøtz tonometer) and visual acuity was finger counting at 1 metre. The best corrected visual acuity was $6 / 60$.

In view of the disastrous postoperative course in the left eye and good visual acuity with correction of aphakic portion in the right eye, it was decided to adopt non-surgical treatment of the right eye. The aphakic area of the pupil was enlarged nasally by repositioning the pupil by iris photocoagulation. Visual acuity improved to $6 / 9$ with $+11.0 \mathrm{D} . \mathrm{sph} /$ +1.0 D.cyl $135^{\circ}$ (Fig. 2). The patient has been followed up for one year. She retains the same visual acuity, and the IOP is $20 \mathrm{mmHg}$.

\section{CASE 2}

A 35-year-old woman attended the Ophthalmology Outpatient Department of Jipmer Hospital in August 1983 with complaints of defective vision both eyes of two years' duration. She had undergone uneventful intracapsular lens extraction in left eye one year previously with good postoperative vision.

Examination revealed that in right eye the anterior chamber was deep and iridodonesis was present. The pupil was normal in size and reaction and the lens was

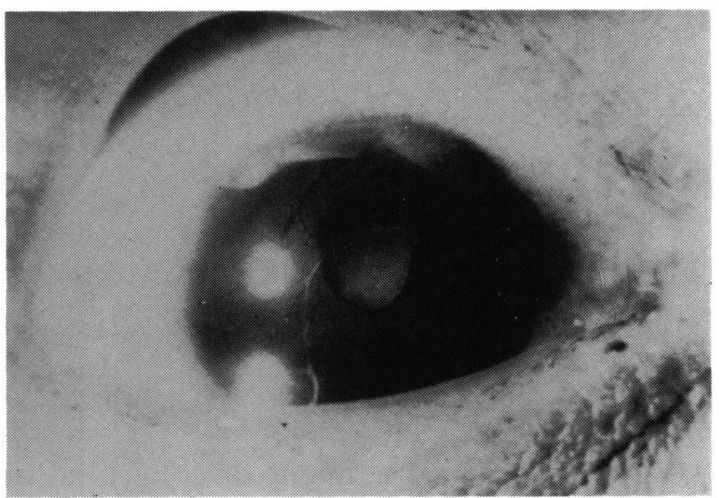

Fig. 3 Upward displacement of pupil with iris photocoagulation.

cataractous, with a visual acuity of counting fingers at 0.5 metre and IOP $17.3 \mathrm{mmHg}$ (Schiøtz tonometer). On dilatation of the pupil the lens was found to be displaced downwards, with good visual improvement after aphakic correction.

The left eye was aphakic, with a peripheral iridectomy. The IOP was $14.6 \mathrm{mmHg}$ (Schiøtz tonometer). The visual acuity improved to $6 / 12$ with $+11.0 \mathrm{D} . \mathrm{sph} /$ $+2 \cdot 0$ D.cyl $165^{\circ}$.

In view of the subluxated lens and good visual improvement with aphakic correction a conservative approach was planned. Iris photocoagulation with xenon arc was done to pull the pupil upwards (Fig. 3). The visual acuity with +12 D.sph was $6 / 9$.

\section{Discussion}

Lens subluxation, a displacement in which the lens remains at least partially within the pupillary area, may occur as a result of heredofamilial disorders, ocular disease, or trauma. Marfan's syndrome commonly causes it and has attracted considerable attention. ${ }^{5}$ Glaucoma is not uncommon in eyes with lens subluxation. The clinical management of subluxated lens is based on awareness of variations in the degree of displacement and an understanding of the adverse effects of this malposition. However, therapy should always be planned to maintain and improve vision.

To accomplish this a number of measures are available. The optical correction of refractive error (phakic) due to irregular astigmatism on account of tilting of the lens is difficult and unsatisfactory; it becomes impossible in the presence of lenticular haze or opacity. Prolonged drug induced mydriasis to permit vision through the aphakic part of the pupil is possible but has the disadvantages of causing glare, drug sensitisation, and the probability of anterior dislocation of the lens. Optical iridectomy may also 
precipitate anterior dislocation, development of pupillary block, or even intraocular infection. Experience with these methods has revealed the limitations of optical correction, prolonged drug induced mydriasis, and the substantial hazards of intraocular surgery. ${ }^{\circ}$

This report describes an additional method of management that is highly beneficial in appropriate cases of subluxation. The method employed is iris photocoagulation to produce a permanent alteration of the pupil and permit vision through the aphakic portion of this expanded aperture (Fig. 3). The determination of the quadrant of iris to be photocoagulated in order to reposition the pupil depends on the position of the displaced lens, and it is evaluated prior to iris photocoagulation by mydriasis and refraction through the aphakic part of pupil.

The pupil can be displaced in the desired direction by iris photocoagulation, presumably owing to iris retraction, and the repositioning of the pupil can be limited to a particular segment. Since there is only localised enlargement of the pupillary aperture, the hazards associated with optical iridectomy or drug induced mydriasis are not encountered. In addition being a non-invasive procedure, the hazards of intraocular surgery are obviated. This procedure can be undertaken in the outpatient department and it is of value even for patients who are otherwise poor surgical risks ${ }^{7}$ or who refuse surgery.

\section{References}

1 Jarret WM. Dislocation of lens. Arch Ophthalmol 1967; 78: 289-93.

2 Barraquer J. Surgery of dislocated lens. Ophthalmology (Rochester) 1972; 76: 44-59.

3 Straatsma BR, Allen RA, Pettit TH, Hall MO. Subluxation of lens treated with iris photocoagulation. Am J Ophthalmol 1966; 61: 1312-24.

4 Peyman GA, Raichard ML, Goldberg MF, Ritacca D. Management of subluxated and dislocated lenses with the vitrophage. $\mathrm{Br}$ J Ophthalmol 1979; 63: 771-8.

5 Francois J. Heredity in ophthalmology. St Louis: Mosby, 1961.

6 Chandler PA. Treatment in dislocation of lens. Arch Ophthalmol 1964; 71: 765.

7 Lamba PA, Santosh KD. Repositioning of pupil for visual disability, Indian J Leprosy 1984; 56: 4-9. 